\title{
INFLUENCIA DEL TRATAMIENTO UV-C SOBRE CARAMBOLA (Averrhoa carambola L.) MÍNIMAMENTE PROCESADA.
}

\author{
María José Andrade', Carlota Moreno
}

\begin{abstract}
RESUMEN
La carambola (Averroha carambola L.) es una fruta tropical exótica, perteneciente a la familia de las oxalidáceas, muy cotizada en los mercados internacionales, conocida como "fruta-estrella" o "starfruit". Su cultivo fue introducido hace aproximadamente unos treinta años en el Ecuador. Los frutos se cosechan verdes, son altamente perecederos, se recomienda su conservación a temperaturas no menores a $5^{\circ} \mathrm{C}$. Tiene una forma ovalada, alargada, con cinco aristas que al corte forman una estrella de cinco puntas por lo que se utiliza en la decoración de la cocina gourmet. El objetivo del presente estudio fue evaluar la influencia del tratamiento UV-C sobre la calidad de carambola mínimamente procesada almacenada a $5^{\circ} \mathrm{C}$. Frutos recién cosechados, lavados y seleccionados, se cortaron en rodajas de $5 \mathrm{~mm}$ de ancho, se dividieron en dos grupos: frutos tratados $\left(13 \mathrm{~kJ} / \mathrm{m}^{2}\right)$ y no tratados (controles) y se almacenaron en bandejas plásticas cubiertas con film PVC durante 21 días. A los 7, 14 y 21 días se determinó la pérdida de peso, acidez, $\mathrm{pH}$, sólidos solubles y se observó visualmente el avance de pardeamiento, decaimiento y firmeza al tacto. Los frutos tratados y controles presentaron un incremento en la pérdida de peso durante el almacenamiento, sin embargo, ésta fue menor en los frutos tratados. No se observaron diferencias significativas en los parámetros físico-químicos analizados a lo largo del almacenamiento. A los 14 días, los frutos control mostraron mayor pardeamiento y pérdida de firmeza que los tratados. Además, a este tiempo, los frutos control dejaron de ser consumibles dado que mostraban desarrollo de microorganismos. Los frutos tratados recién mostraron desarrollo fúngico a los 21 días de almacenamiento a $5^{\circ} \mathrm{C}$. Los resultados sugieren que el tratamiento UV-C retardó los síntomas de daño permitiendo la conservación de carambola mínimamente procesada con una buena calidad comercial por más tiempo.
\end{abstract}

\footnotetext{
${ }^{1}$ Universidad Tecnológica Equinoccial, Facultad de Ciencias de la Ingeniería, Av. Occidental y Mariana de Jesús. Quito, Ecuador.acmj2221@ute.edu.ec
} 


\section{INTRODUCCIÓN}

La carambola (Averrhoa carambola L.) es una fruta exótica muy cotizada en los mercados internacionales, conocida popularmente como "fruta estrella" o "star fruit". Pertenece a la familia de las Oxalidáceas. En función de su procedencia, recibe distintos nombres: en República Dominicana, "cinco dedos”; en Costa Rica, "tiriguro"; en Brasil, "caramboleiro" y en Venezuela, "tamarindo chino" o "tamarindo dulce", de gran empleo en la decoración de diversos platos exquisitos (Eroski, s.f.).

La carambola o fruta china tiene forma ovalada, alargada, con cinco aristas o alas y, al corte, de estrella de cinco puntas. Es de pequeño tamaño, con una longitud que oscila entre 7 y 12 centímetros, tiene una piel fina, lustrosa y comestible, de color entre verde o dorado y amarillo-anaranjado cuando está madura. La pulpa es crujiente, de suave textura y amarilla vidriosa, la pulpa tiene pocas o ninguna semilla, es abundante, crujiente, jugosa y con un fino sabor agridulce.

En Ecuador, la carambola se cultiva principalmente en Quinindé, Santo Domingo, Calceta, La Concordia y la Maná. El rendimiento de producción promedio es de 28000 - $32000 \mathrm{~kg} / \mathrm{Ha}$ (SIGAGRO, 2002).

Es preferible conservarla en un lugar fresco, lejos del contacto directo con la luz del sol. Si al comprarla aún está verde, se debe dejar a temperatura ambiente $\left(20^{\circ} \mathrm{C}\right)$. Una vez madura, se recomienda guardar la carambola en la nevera, donde se conserva en óptimas condiciones hasta dos o tres semanas a una temperatura no inferior a $5^{\circ} \mathrm{C}$ (SICA, s.f.).

Productos mínimamente procesados.- Las frutas y hortalizas mínimamente procesadas en fresco, también denominados comercialmente de la IV Gama, constituyen un sector de rápido crecimiento en la industria de los alimentos. Tratándose de productos muy perecederos, deben ser manipulados siguiendo estrictas normas de control de calidad. Esto debido al cambio notable en los hábitos alimentarios de la población

Previo al procesado mínimo se realizan distintas operaciones unitarias que, de forma general, se pueden resumir en las siguientes: selección del cultivar a procesar, elección del grado de madurez óptimo, clasificación y acondicionamiento. Luego continúan las operaciones propias del procesamiento mínimo: lavado del producto entero, deshojado, pelado, deshuesado, cortado, lavado y desinfectado. Una vez que los productos se procesan, se empaquetan en bolsas selladas o en bandejas cubiertas con plásticos, con o sin atmósfera modificada para, posteriormente, ser almacenados y transportados bajo refrigeración (Lobo y González, s.f.). 
Estos productos son, por lo general, más perecederos que los productos intactos de los que proceden siendo, en general, su vida útil entre siete y diez días. Las modificaciones de calidad más importantes que sufren se deben a la presencia de superficies cortadas y tejidos vegetales dañados, a que dicho proceso no puede asegurar la esterilización o la estabilidad microbiológica del producto y a que, su metabolismo sigue estando activo. Así, las reacciones de degradación que se producen afectan a cualidades organolépticas tales como el color, firmeza, aroma, sabor y valor nutricional haciéndolos más susceptibles a perder su calidad higiénico-sanitaria (Lobo y González, s.f.).

En los últimos años se han aplicado tecnologías postcosecha combinadas como atmósfera modificada junto con la aplicación de ozono, 1-MCP, ácido absícico, radiación gamma, radiación UV-C, etc.

Por otro lado, numerosos estudios han demostrado la efectividad del tratamiento con radiación UV-C en productos IV GAMA de mango (González-Aguilar y col., 2007), melón (Lamikanra y col., 2005), tuna (Piga y col; 1998), brócoli (Costa y col., 2006), pimiento (Vicente y col., 2005), entre otros.

Radiación ultravioleta (UV-C).- Es la porción más energética del espectro electromagnético de la luz comprendida entre los 290 y $100 \mathrm{~nm}$ y posee una importante acción bactericida.

Actualmente, el uso de muchos tratamientos poscosecha con sustancias químicas causa problemas ecológicos o son potencialmente perjudiciales para el ser humano por lo que en muchos países se ha restringido su uso. Es necesario entonces, desarrollar métodos de control alternativos y seguros (Artés, 1995) como el uso de la radiación con luz UV-C como posible estrategia en la tecnología de poscosecha, basándose en el concepto de "hormesis" (Luckey, 1980), definido como la estimulación de un efecto beneficioso en los tejidos en respuesta a dosis bajas o subletales de un agente causante de estrés físico o químico.

Tratamientos que usan la irradiación UV-C, han sido probados como una alternativa tecnológica capaz de reducir la tasa de maduración y activar una respuesta de defensa natural en la planta para incrementar la vida poscosecha de frutos y hortalizas, por ejemplo Liu y col. (1991) reportaron que la exposición a dosis bajas de UV-C retrasó la maduración y senescencia en manzana, encontrándose resultados similares en otros productos como mango (González-Aguilar y col., 2001), durazno (González-Aguilar y col., 2004) y naranja (D’hallewin y col., 1999); sin embargo pocos estudios se han realizado para elucidar el modo de acción de la radiación UV-C sobre estos sistemas. Barka y col., (2000) estudiaron los efectos de la luz UV-C en la inducción de algunas enzimas en tomate y 
encontraron una significativa peroxidación lipídica, pérdida de electrolitos a través de membrana y la inducción de enzimas relacionadas al estrés oxidativo como guayacol peroxidasa y ascorbato peroxidasa, además reportaron la activación de enzimas involucradas en mecanismos de defensa como la fenilalanina amonioliasa y lipooxigenasa (Barka, 2001).

La radiación UV-C se perfila como una de las tecnologías con mayor aplicación en el futuro. Una propuesta de Cisneros-Zevallos (2003), sugiere la aplicación poscosecha de un tipo de estrés abiótico controlado (exposición a luz UV-C) para inducir la producción e incremento de la síntesis de compuestos fotoquímicos con actividad nutracéutica, o la reducción de compuestos indeseables. Así el control del estrés inducido por la luz UV-C puede usarse como una herramienta para reforzar las propiedades benéficas de productos frescos enteros o cortados.

Con estos antecedentes, se hace necesaria más evidencia experimental para elucidar el modo de acción de esta tecnología aplicada a productos hortifrutícolas.

\section{MATERIALES Y MÉTODOS}

Tratamiento con luz UV-C y selección de la dosis efectiva.- Para determinar las condiciones experimentales adecuadas, se ensayaron diferentes dosis de luz UV-C. Frutos recién cosechados, lavados y seleccionados, se cortaron en rodajas de $5 \mathrm{~cm}$ de ancho, se dividieron se dividieron en dos grupos: control (no irradiados) y tratados (irradiados). Estos últimos se colocaron bajo cuatro lámparas UV-C (TUV G30T8, 30W, Philips) a una distancia de $30 \mathrm{~cm}$ y fueron irradiados con dosis de 6; 10 y 13 $\mathrm{kJ} / \mathrm{m}^{2}$. La intensidad de la radiación fue medida con un radiómetro digital UV (UVX RADIOMETER UVP).

Cada rodaja fue rotada para asegurar una exposición uniforme a la luz UV en las superficies anversa y reversa. Finalizado el tratamiento los frutos se colocaron en bandejas plásticas cubiertos con un film de PVC y se almacenaron a $5^{\circ} \mathrm{C}$ durante 21 días. Los controles (sin ningún tratamiento) se empacaron con la misma película y almacenaron directamente a $5^{\circ} \mathrm{C}$. Tres bandejas de frutos control y tratados se retiraron de la cámara de almacenamiento a los 0, 7, 14 y 21 días para evaluar el efecto de cada tratamiento sobre el índice de daño y pérdida de peso. La experiencia completa se realizó por duplicado.

Una vez elegida la dosis de $13 \mathrm{~kJ} / \mathrm{m}^{2}$, se efectuó el tratamiento sobre rodajas de carambola (como se indicó anteriormente) y se los almacenó junto a los frutos control a $5^{\circ} \mathrm{C}$ por $0,7,14$ y 21 días. A cada 
día de muestreo las bandejas fueron retiradas de la cámara de almacenamiento y luego de estimar el índice de daño, pérdida de peso además de realizar el recuento de mohos y aerobios totales. El tejido restante se cortó y congeló en $\mathrm{N}_{2}$ líquido para finalmente conservarlo a $-18^{\circ} \mathrm{C}$ para su posterior análisis bioquímico.

Pérdida de peso: se pesó cada bandeja al inicio y al término de cada período de almacenamiento. Se determinó la pérdida de peso como porcentaje del peso inicial.

Sólidos solubles totales: Se determinaron en el jugo de carambola extraído, mediante un refractómetro de mano marca Atago.

pH: Se midió en el jugo de carambola de cada muestra, con un potenciómetro digital marca Metter Toledo 320.

Acidez titulable como \% Ácido cítrico: $5 \mathrm{~mL}$ de jugo de extracto de jugo de carambola se titularon 5 $\mathrm{mL}$ con $\mathrm{NaOH}(0,1 \mathrm{~N})$, utilizando fenolftaleína $(0,1 \%)$ como indicador.

Índice de madurez: se obtuvo de la relación de los sólidos solubles y de la acidez titulable.

Índice de daño: Cada día de muestreo los frutos se evaluaron visualmente utilizando una escala subjetiva, considerando los siguientes parámetros: decaimiento, pardeamiento y firmeza al tacto. Usando índice de daño (ID) con una escala subjetiva de 1 a 4, donde: 4=daño severo, 3=daño moderado, $2=$ daño ligero y $1=\sin$ daño.

Análisis estadístico: En todas las experiencias se empleó un diseño completamente al azar con un solo factor. Los resultados fueron procesados mediante un ANOVA y las medidas comparadas por el test LSD con una significancia de 0,05 usando el software STATGRAPHICS versión 4.0 


\section{RESULTADOS Y DISCUSIÓN}

Selección de la dosis.- La pérdida de peso (Tabla 1) de los frutos control fue de 3,8\% con respecto al día inicial del almacenamiento. En los frutos tratados con las dosis de 6 y $10 \mathrm{~kJ} / \mathrm{m}^{2}$, fue 8,4 y $9,8 \%$ respectivamente, mientras que los frutos tratados con $13 \mathrm{~kJ} / \mathrm{m}^{2}$ sufrieron la menor pérdida de peso $(4,7 \%)$.

Tabla 1. Análisis microbiológicos, pérdida de peso (\%), e índice de daño en frutos control y tratados $\left(6,10\right.$ y $\left.13 \mathrm{~kJ} / \mathrm{m}^{2}\right)$ durante 21 días de almacenamiento a $5^{\circ} \mathrm{C}$.

\begin{tabular}{|c|c|c|c|c|c|c|}
\hline \multirow{2}{*}{ Parámetro } & & \multirow[t]{2}{*}{ Tiempo (Días) } & \multirow[t]{2}{*}{ Control } & \multicolumn{3}{|c|}{ Dosis UV-C } \\
\hline & & & & $6 \mathrm{~kJ} / \mathrm{m}^{2}$ & $10 \mathrm{~kJ} / \mathrm{m}^{2}$ & $13 \mathrm{~kJ} / \mathrm{m}^{2}$ \\
\hline \multirow{5}{*}{ Análisis microbiológicos } & Mohos & 0 & 3,1 & 3,4 & 1,4 & 1,0 \\
\hline & $(\log \mathrm{PM} / \mathrm{g})$ & 21 & 4,7 & 5,9 & 4,7 & 4,5 \\
\hline & Aerobios & 0 & 3,4 & 2,6 & 4,5 & 4,5 \\
\hline & totales & 21 & 6,6 & 4,5 & 4,7 & 4,7 \\
\hline & $(\log \mathrm{FC} / \mathrm{g})$ & & & & & \\
\hline Pérdida peso (\%) & & 21 & 3,8 & 8,4 & 9,8 & 4,7 \\
\hline Índice de Daño & & 21 & 3,7 & 2,3 & 2,5 & 2,6 \\
\hline
\end{tabular}

También se encontró que los frutos control presentaron síntomas de daño a los 7 días de almacenamiento, diferenciándose de los frutos tratados que mantenían una mejor calidad. Aún a los 14 días de almacenamiento los frutos tratados presentaron mejores características con respecto a los frutos control (tabla 1). A los 21 días de almacenamiento, los frutos control habían perdido totalmente su calidad alcanzando un valor de ID de 3,7; en cambio, los frutos tratados con 6,10 y $13 \mathrm{~kJ} / \mathrm{m}^{2}$ alcanzaron valores de ID de 2,6; 2,5 y 2,3 respectivamente (tabla 1). En cuanto al análisis microbiológico, las dosis de 10 y $13 \mathrm{~kJ} / \mathrm{m}^{2}$ retrasaron el crecimiento de mohos y aerobios totales con respecto a la dosis de $6 \mathrm{~kJ} / \mathrm{m}^{2}$ y las muestras control (datos no mostrados). Por lo tanto, la dosis de 13 $\mathrm{kJ} / \mathrm{m}^{2}$ fue seleccionada para los ensayos posteriores.

Efecto del tratamiento UV-C sobre el índice de daño.- Habiendo seleccionado una dosis de 13 $\mathrm{kJ} / \mathrm{m}^{2}$ se evaluó el desarrollo del índice de daño (ID). En la figura 1, se expone la variación hallada en los ID de los frutos en función del tiempo de almacenamiento. 


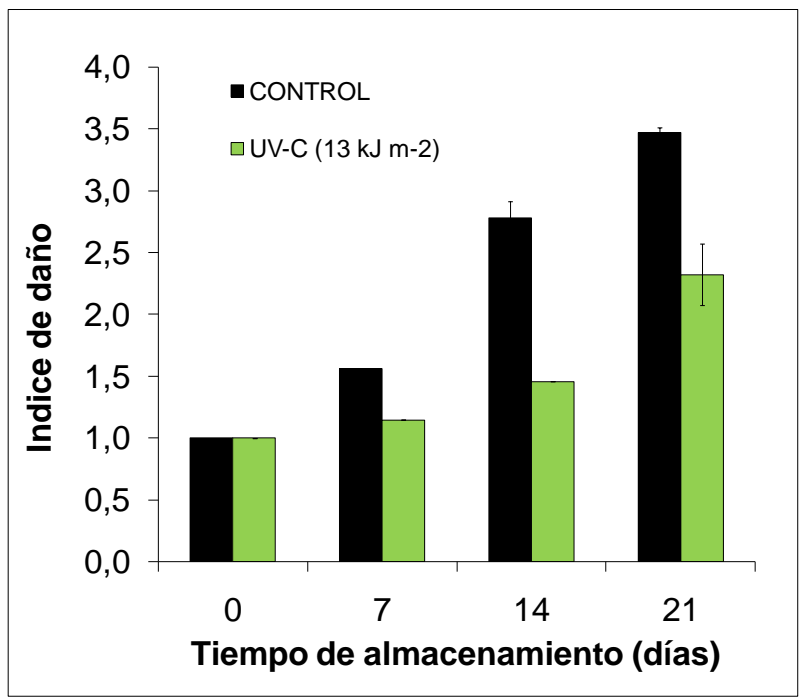

Figura 1. Variación del índice de daño de carambola fresca cortada en función del tiempo de almacenamiento a $5^{\circ} \mathrm{C}$.

Se evidenció daño en los frutos control a partir del día 7, alcanzando un daño moderado a severo $(\mathrm{ID}=3,5)$ en el día 21 perdiendo totalmente su calidad organoléptica y comercial, mientras que los frutos tratados alcanzaron al final del almacenamiento un daño de leve a moderado (ID= 2,7) manteniendo una mejor calidad organoléptica y comercial (Figura 2).

Efecto del tratamiento UV-C sobre la pérdida de peso.- Se evaluó la pérdida de peso al final de cada tiempo de almacenamiento.

Los frutos tratados presentaron un comportamiento similar a los controles pero aumentaron la pérdida de peso en menor proporción.
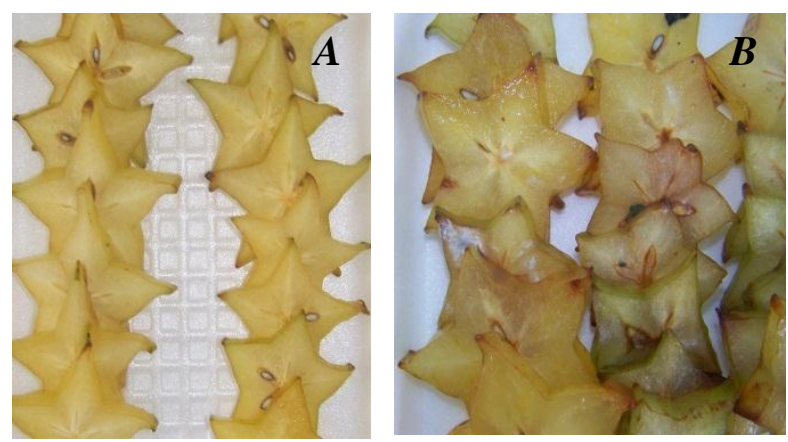

Figura 2. Desarrollo del índice de daño en frutos de carambola fresca cortada tratada (A) con $13 \mathrm{~kJ} / \mathrm{m}^{2}$ y controles (B) luego de 21 días de almacenamiento a $5^{\circ} \mathrm{C}$. 
Efecto del tratamiento UV-C sobre los parámetros físico-químicos.- No se observaron diferencias significativas en los parámetros físico-químicos analizados a lo largo del almacenamiento (Tabla 2). El índice de madurez aumentó durante el período de ensayo. A los 14 días, los frutos control mostraron mayor pardeamiento y pérdida de firmeza que los tratados.

Tabla 2. Parámetros físico-químicos en frutos de carambola fresca cortada tratada (UV-C) con $13 \mathrm{~kJ} / \mathrm{m}^{2}$ y controles luego de 21 días de almacenamiento a $5^{\circ} \mathrm{C}$.

\begin{tabular}{|c|c|c|c|c|c|}
\hline & $\begin{array}{l}\text { Tiempo } \\
\text { (días) }\end{array}$ & $\begin{array}{l}\text { Sólidos } \\
\text { solubles }\end{array}$ & $\mathrm{pH}$ & $\begin{array}{l}\text { Acidez titulable } \\
\text { (\% Ácido Cítrico) }\end{array}$ & $\begin{array}{l}\text { Índice de } \\
\text { madurez }\end{array}$ \\
\hline \multirow{2}{*}{0} & Control & $7,1 \pm 0,1$ & $3,74 \pm 0,04$ & $0,38 \pm 0,01$ & $18,41 \pm 0,49$ \\
\hline & UV-C & $7,2 \pm 0,3$ & $3,68 \pm 0,09$ & $0,37 \pm 0,03$ & $19,56 \pm 2,39$ \\
\hline \multirow{2}{*}{7} & Control & $7,1 \pm 0,3$ & $3,95 \pm 0,07$ & $0,35 \pm 0,01$ & $20,17 \pm 1,62$ \\
\hline & UV-C & $7,4 \pm 0,4$ & $3,84 \pm 0,22$ & $0,36 \pm 0,01$ & $20,60 \pm 0,87$ \\
\hline \multirow{2}{*}{14} & Control & $7,0 \pm 0,2$ & $3,67 \pm 0,05$ & $0,33 \pm 0,01$ & $21,32 \pm 0,72$ \\
\hline & UV-C & $7,0 \pm 0,1$ & $3,68 \pm 0,13$ & $0,32 \pm 0,02$ & $21,71 \pm 1,90$ \\
\hline \multirow{2}{*}{21} & Control & $6,6 \pm 0,2$ & $3,57 \pm 0,12$ & $0,32 \pm 0,03$ & $20,78 \pm 2,15$ \\
\hline & UV-C & $7,0 \pm 0,4$ & $3,67 \pm 0,10$ & $0,30 \pm 0,02$ & $22,90 \pm 1,39$ \\
\hline
\end{tabular}

Efecto de la radiación UV-C sobre el desarrollo fúngicos.- Como se puede observar en la figura 3, partir del día 14 los frutos control dejaron de ser consumibles dado que mostraban desarrollo de microorganismos.

Los frutos tratados recién mostraron desarrollo fúngico a los 21 días de almacenamiento a $5^{\circ} \mathrm{C}$. 


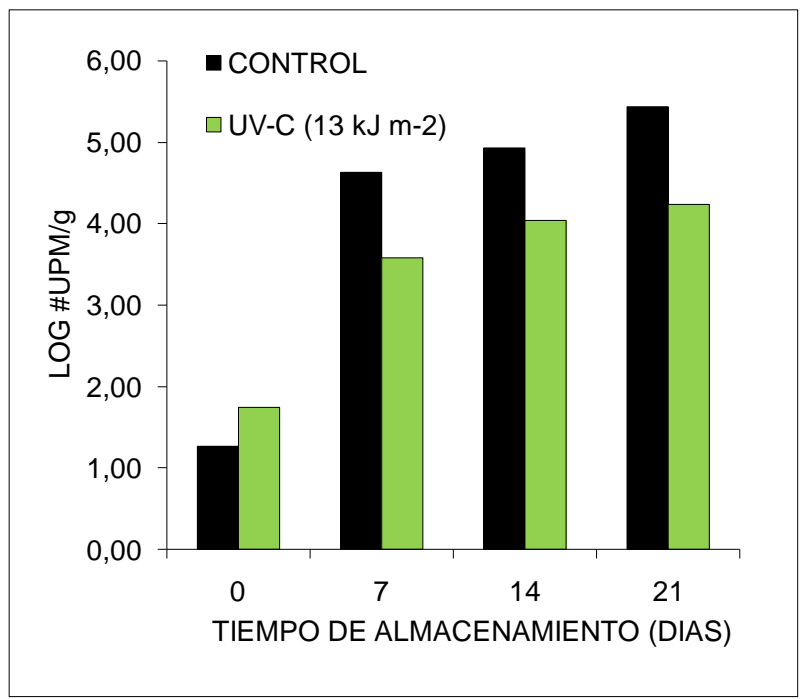

Figura 3. Decaimiento en frutos control y tratado a lo largo del almacenamiento refrigerado $5^{\circ} \mathrm{C}$.

\section{CONCLUSIÓN}

La aplicación de la radiación UV-C redujo el decaimiento en carambola mínimamente procesada. La dosis de $13 \mathrm{~kJ} / \mathrm{m}^{2}$ retrasó los síntomas de decaimiento durante 21 días.

El uso de la radiación UV-C en combinación con refrigeración podría utilizarse como una tecnología para extender la vida útil de carambola mínimamente procesada, sin embargo, son necesarias futuras investigaciones para entender el mecanismo de acción de la radiación sobre este fruto.

Los resultados de estos estudios servirán de base para iniciar el trabajo en esta línea de investigación en la Carrera de Ingeniería de Alimentos de la Facultad de Ciencias de la Ingeniería.

\section{BIBLIOGRAFÍA}

Artés, F. Innovaciones en los tratamientos físicos modulados para preservar la calidad de los productos hortofrutícolas en la postrecolección. I Pretratamientos térmicos. Rev. Esp. Ciencia Tecnol. Alim. No. 35; 1995: 45-64, 35, 139-149 у 35, 247-269

Barka, E. A. Protective enzymes against reactive oxygen species during ripening of tomato .Lycopersicon esculentum L.. fruits in response to low amounts of UV-C. Aust. J. Plant Physiol. No. 28; 2001:785-791 
Barka, E. A., Kalantari, S., Makhlouf, J. y Arul, J. Effects of UV-C irradiation on lipid peroxidation markers during ripening of tomato .Lycopersicon esculentum L.. fruits. Aust. J. Plant Physiol. No. 27; 2000: 147-152.

Cisneros-Zevallos, L. The use of controlled postharvest abiotc stresses as a tool for enhancing the nutraceutical content and adding-value of fresh fruits and vegetables. J. of Food Sc. No. 68; 2003: $1560-1564$

Costa, L., Vicente, A. R., Civello, P. M., Chaves, A. R. y Martínez, G. A. UV-C treatment delays postharvest sescence in brócoli florets. Posth. Biol. and Techn. No. 39; 2006: 204-210

D’hallewing G., Schirra, M., Manueddu, M., Piga, S. y Ben-Yehoshua, S. Scoparone and scopoletin accumulation and ultraviolet- $\mathrm{C}$ induced resistance to postharvest decay in oranges as influenced by harvest date. J. Am. Soc. Hort. Sci. No. 124; 1999: 702-707.

Eroski, Fundación. www.Carambola Guía de Frutas CONSUMER_es EROSKI.com.

González-Aguilar, G. A., Wang, C. y Buta, G. UV-C irradiation reduces breakdown and chilling injury of peaches during cold storage. Jour. of fhe Sc. Of Food and Agric. 2004. 84 .5. 415-442

González-Aguilar, G., Zavaleta-Gatica, R. y Tiznado-Hernández, M.E. Improving postharvest quality of mango 'Haden' by UV-C treatment. Posth. Biol. And Techn.2007. 45 .1. 108-116

González-Aguilar, G.A., Wang, C.Y., Buta, J.G. y Krizek, D.T. Use of UV-C irradiation to prevent decay and maintain postharvest quality of ripe "Tommy Atkins" mangoes. Int. J. Food Sci. Tech. 2001. $36,767-773$

Lamikanra, O., Kueneman, D., Ukuku, D. y Bett-Garber, K. Effect of Processing Under Ultraviolet Light on the Shelf Life of Fresh-Cut Cantaloupe Melon. Journal of Food Science. 70; 2005: 534-539

Liu, J.; Stevens, C.; Khan, V.A. y Kabwe, M. (1991). The effect of ultraviolet irradiation on shelf-life and ripening of peaches and apples. J. Food Qual. 14, 299-305

Lobo, M., y González, M. (Sin fecha). Estado actual de los productos mínimamente procesados en España. Laboratorio de Fisiología Vegetal .Dpto. Fruticultura Tropical., Instituto Canario de Investigaciones Agrarias, La Laguna, Tenerife, Islas Canarias, España.

Luckey, T.D. (1980). Hormesis with ionizing radiation, CRC press, Boca Raton.

Piga, A., D’hallewin, G., D’aquino, S. y Agabbio, M. Influence of film wrapping and UV irradiation on cactus pear quality after storage. Packaging Technology and Science. 10; 1998: 59-68 
SICA.[sin fecha]. Carambola Fruta china Star fruit Averroha carambola L.Serivicios de información de censos agropecuarios SICA. http://www.sica.gov.ec

SIGAGRO. (2002). Ecuador Estimación de la producción. Frutas, oleaginosas, fibras, cabuya, bebidas, té y otros cultivos. http://www.sica.gov.ec/agro/docs/CUADRO.htm

Vicente, A., Pineda, C., Lemoine, L., Civello, P., Martínez, G., Chaves, A. UV-C treatments reduce decay, retain quality and alleviate chilling injury in pepper. Postharvest Biol. Technol. 35; 2005: 69-79. 